\title{
DEVELOPMENT AND EVALUATION OF INDIRECT HEMAGGLUTINATION ANTIBODY TEST (IHAT) FOR SEROLOGICAL DIAGNOSIS AND SCREENING OF BOVINE CYSTICERCOSIS IN ETHIOPIA
}

\author{
Nigatu Kebede ${ }^{1}$, Getachew Tilahun ${ }^{1}$ and Asrat Hailu ${ }^{2}$ \\ ${ }^{1}$ Aklilu Lemma Institute of Pathobiology, Addis Ababa University, PO Box 1176 \\ Addis Ababa, Ethiopia. E-mail: knigatu2003@yahoo.com \\ ${ }^{2}$ Department of Microbiology, Immunology and Parasitology, Faculty of Medicine, \\ Addis Ababa University, PO Box 1176, Addis Ababa, Ethiopia
}

\begin{abstract}
The study was conducted to develop and evaluate an Indirect Hemagglutination Antibody Test (IHAT) for the serological diagnosis of Cysticercus bovis in live animals. IHAT was set-up inhouse and used to test serum samples of cattle against sheep red blood cell (SRBC) coated with crude extracts of $C$. bovis cyst. Serum samples for screening were collected from Addis Ababa abattoir $(n=522)$, a dairy farm from the suburbs of Addis Ababa $(n=101)$, and a traditional extensive farm $(n=109)$. Postmortem inspection was conducted on a total of 522 animals slaughtered in Addis Ababa Abattoir, out of which $39(7.5 \%)$ were positive for C. bovis. Thirty-seven of these had viable C. bovis cysts. IHAT titres were determined from serially diluted sera, with a cut-off value set at a titre of 1:64. Cross reaction with other helminths was either absent or very low. Sensitivity and specificity of the IHAT were $100 \%$ and at least $90 \%$, respectively. Based on this test, 149 cattle $(28.5 \%)$ from the Abattoir, 33 cattle (30.3\%) from the extensive farm and 8 cattle $(7.9 \%)$ from the dairy farm were positive for $C$. bovis. The difference in prevalence between the dairy farm and extensive farm was significant $(\mathrm{P}<0.05)$. Further refinement and improvement of the test is necessary to increase the diagnostic potential. The test is recommended for use in large scale serological screening and mapping of C. bovis infection in major animal husbandry locations in Ethiopia.
\end{abstract}

Key words/phrases: Body condition, Cysticercus bovis, Ethiopia, IHAT, postmortem inspection

\section{INTRODUCTION}

Bovine cysticercosis is caused by the larval stage of the tapeworm Taenia saginata. Humans are the final hosts of the parasite. Infection in man is acquired by ingestion of raw or undercooked beef containing the larval cysts, Cysticercus bovis, while cattle become infected by ingesting tapeworm eggs passed with human feaces (Hancock et al., 1989). The life cycle and transmission of T. saginata occurs most commonly in environments characterized by poor sanitation, poor livestock husbandry practices and inadequate meat inspection and control. The beef tapeworm is found almost all over the world, albeit at very low prevalence in developed countries. Moderate prevalence levels are seen in southern Asia. High prevalence rates occur in subSaharan Africa, especially in Eastern Africa where it causes an important economic loss due to condemnation of meat (Cabaret et al., 2002).

Cysticercosis is more prevalent in feedlots and in traditional farming systems than in dairy farms. It has been suggested that the continuous man to animal contact and the involvement of causal workers in feedlots may be factors that contribute to $T$. saginata transmission (Dorny et al., 2002).
In many developing countries, this disease constitutes a serious but sometimes less recognized public health problem (Minozzo et al., 2002). In Ethiopia, high prevalence rates of $89.41 \%$ (Tembo, 2001) have been reported in different agro-climatic zones in humans. This high prevalence was associated with the habit and/or culture of eating raw or undercooked beef, which are the source of T. saginata infection in man (Gebre Emanuel Teka, 1997). In cattle, reported prevalence rates of $C$. bovis in Ethiopia ranged from 2.2\% to 3.2\% (Teka Gebre Emanuel Teka, 1997; Tembo, 2001).

Although Ethiopia is ideally placed to export live animals to the big markets of the Middle East and North and West Africa, export earnings are relatively low. This is mainly due to the presence of a number of animal health problems, including bovine cysticercosis (EARO, 2000). It is, therefore, important that due attention be given to this disease to improve the quality and quantity of beef so as to satisfy the domestic consumption and to increase the foreign export revenue.

The objective of the current study was to evaluate the performance of IHAT in the diagnosis and large-scale serological screening of $C$. bovis infection in cattle with the view of replacing the 
conventional parasitological methods, which are inaccurate and cumbersome.

\section{MATERIALS AND METHODS}

\section{Study areas and test samples}

The study was conducted on samples from Addis Ababa Abattoir $(n=522)$, Repi PLC Dairy Farm $(n=101)$ and Yeka-Abado Farmers Association $(n=109)$. Cattle slaughtered in Addis Ababa Abattoir were brought from different regions in the country. In Repi PLC Dairy Farm the animals are mostly house fed with occasional grazing in the restricted farm area. Replacement stock is bred in the farm and the farm employs permanent personnel. At Yeka-Abado Farmers Association grazing and watering sites are communal. There are no latrines in the area, bush defecation is practiced and animals often get in contact with human faeces. Focal and blood samples were collected from all 732 cattle. Blood samples were taken from the study animals and the serum separated using the methods described earlier (Dorny et al., 2002) and kept at $-20^{\circ} \mathrm{C}$ until tested. Sera of cattle with confirmed C. bovis cyst, originating from Addis Ababa slaughterhouse, were used as positive controls. Negative control sera $(n=11)$ were obtained from Department of Parasitology, Toulouse, France.

\section{Postmortem inspection}

A total of 522 adult cattle (514 males and 8 females) slaughtered at Addis Ababa abattoir were subjected to postmortem inspection. Carcasses of these animals were thoroughly inspected. For incisions and inspection, the procedures outlined by Ministry of Agriculture (MoA, 1972) were adhered to.

\section{Faecal examination}

The purpose of faecal examination was to evaluate the presence of cross reaction with other gastrointestinal parasites. Faecal samples were collected directly from the rectum and taken to the laboratory with tightly closed universal bottles and processed using the standard methods of sedimentation and flotation (Sloss et al., 1994; Kaufmann, 1998).

\section{IHAT: antigen preparation}

Cysticercus bovis infected tissues/organs were collected from Addis Ababa Abattoir. Cysts were then dissected out from the muscles, placed in a petri-dish, and washed intensively with physiological saline. For preparation of crude antigen, the intact cysts were ground using sterile mortar and pestle. A small amount of distilled water was added and the material further homogenized using a tissue grinder and ultrasonicator at 3 cycles for 2 minutes each, at $1 \mathrm{~mA}$ and $20 \mathrm{kHz}$. The homogenate was centrifuged at a speed of $10,000 \mathrm{rpm}$ for 35 minutes at $4^{\circ} \mathrm{C}$. The supernatant and sediment were separated, placed into different Nunc cryo tubes and stored in liquid nitrogen. Protein concentrations of the crude antigen extracts were determined using the Lowry method (Lowry et al., 1951), and appropriate concentrations of the antigens were used to coat formalinized sheep red bloods (SRBCs) (Yamamoto et al., 1991).

\section{Sensitization and coating of sheep red blood cell}

One hundred $\mu \mathrm{l}$ of $25 \%$ glutaraldehyde was mixed with $0.5 \mathrm{ml}$ packed SRBCs in $10 \mathrm{ml}$ phosphate buffer saline (PBS) (pH 7.2). This mixture was incubated at $37^{\circ} \mathrm{C}$ water bath for 20 minutes; and subsequently centrifuged at $2000 \mathrm{rpm}$ for 5 minutes at $4^{\circ} \mathrm{C}$. The supernatant was discarded, and the pelleted SRBC was re-suspended in $10 \mathrm{ml}$ PBS (pH 7.2) and washed twice with $10 \mathrm{ml}$ PBS (pH 7.2) (Yamamoto et al., 1991).

Coating of SRBC with antigen involved gentle mixing of $250 \mu \mathrm{l}$ antigen (stock concentration of 5.0 $\mathrm{mg} / \mathrm{ml}$ ) to $0.5 \mathrm{ml}$ sensitized SRBCs in a total volume of $4.0 \mathrm{ml}$ PBS (pH 6.4). The mixture was incubated at $37^{\circ} \mathrm{C}$ in a water bath for 30 minutes (shaking twice). This mixture was centrifuged and the pellet washed three times with PBS ( $\mathrm{pH}$ 7.2) containing 1\% Fetal Calf Serum (FCS), and finally suspended in a total volume of $10.0 \mathrm{ml}$ PBS (pH 7.2) containing $1 \%$ FCS, which gave $5 \%$ suspension of antigen coated SRBCs ready to use for the test (Shirai et al., 1975; Yamamoto et al., 1991; Ferreira et al., 1997).

\section{IHAT: standardization and test procedures}

To determine the detection range and analytical sensitivity of the test method, assays were set-up using positive and negative control sera. The test procedures involved a serial titration of serum samples to determine the highest levels (titres) of hemagglutinating antibodies (Yamamoto et al., 1991). A cut-off titre was set empirically: first by using standard positive and negative sera, and secondly with tests involving 39 sera of cattle with C. bovis infection. Intrinsic validity (sensitivity and specificity) of the test was checked by comparison with parasitological results of postmortem 
inspection. Presence of cross reactivity with hydatidosis and other helminths were also checked in tests of serum samples originating from cattle with confirmed infections with gastrointestinal helminthiasis.

To V-shaped Griener microtitre plates, $50 \mu$ PBS ( $\mathrm{pH}$ 7.2) was distributed to each well except wells in the $2^{\text {nd }}$ column. $100 \mu \mathrm{l}$ of test sera diluted $1 / 8$ separately in PBS ( $\mathrm{pH} 7.2)$ were added to the $2^{\text {nd }}$ column. Serial double-fold dilutions of sera were made by transferring $50 \mu \mathrm{l}$ the $1 / 8$ diluted serum to a series of wells containing $50 \mu$ l of PBS (pH 7.2). Finally $50 \mu$ l of $5 \%$ antigen coated SRBCs was added to the respective wells and kept on the bench undisturbed for at least $3 \mathrm{hrs}$ (plates could be read after overnight incubation at room temperature). The settling patterns of agglutination reactions were used to determine the levels of antibody expressed by hemagglutination titres. The end point titre was determined as the last well with discernible agglutination preceding the well with settling pattern comparable to wells containing control samples (wells in the first column) usually a distinctly compact dot (Yamamoto et al., 1991).

All test sera were first pre-adsorbed prior to testing by mixing equal volumes of test sera and uncoated sensitized SRBCs. The mixture was incubated in water bath at $37^{\circ} \mathrm{C}$ for 20 minutes, and centrifuged at $2000 \mathrm{rpm}$ for 5 minutes. This procedure allowed removing non-specific reactions by group agglutinins.

\section{Data analysis}

The data obtained from faecal examination and IHAT were entered into MS Excel. Statistical analysis of data was accomplished by Epi Info version 6 and SPSS version 11.5. Results obtained from faecal examination were compared with that of IHAT using Chi-square. Diagnostic value of IHAT was determined taking postmortem inspection as gold standard. The cut-off titre of IHAT was determined using hemagglutination titres of serum samples in C. bovis infected and uninfected cattle as discussed by Thrusfield (1995). Chi-square tests were applied to see if statistically significant association exists between IHAT titre and infection of cattle with gastrointestinal helminthes.

\section{RESULTS}

\section{Postmortem inspection}

All the cattle slaughtered at Addis Ababa Abattoir were $98.6 \%$ adult males and $1.4 \%$ adult females. Of the total 522 animals inspected, 39 (7.5\%) animals were positive for $C$. bovis. Thirty-seven $(7.1 \%)$ of the carcasses had viable $C$. bovis cyst whereas the remaining two $(0.4 \%)$ had calcified cysts.

\section{Faecal examination}

To detect gastrointestinal parasites, the flotation and sedimentation techniques were conducted on all faecal samples. The prevalence of parasitosis in the Yeka-Abado Farmers Association (32.1\%) was significantly different from Repi PLC Dairy Farm $(4.95 \%), \mathrm{p}<0.05$. No significant difference was observed $(\mathrm{P}>0.05)$ between faecal examination results of Yeka-Abado Farmers Association and samples of Addis Ababa Abattoir, 32.11\% and $40.23 \%$, respectively. The commonest gastrointestinal and liver parasites identified were Fasciola hepatica, F. gigantica, Trichuris spp., Strongyle spp., and Coccidia (Table 1).

Table 1. Prevalence of gastrointestinal helminthes at the study sites.

\begin{tabular}{|c|c|c|c|c|}
\hline \multirow[b]{2}{*}{ Parasites identified } & \multicolumn{3}{|c|}{ Source of cattle } & \multirow{2}{*}{$\begin{array}{l}\text { (Total } \mathbf{n}=\mathbf{7 3 2} \text { ) } \\
\text { No }(\%) \text { positive }\end{array}$} \\
\hline & $\begin{array}{l}\text { Abattoir }(\mathrm{n}=522) \\
\text { No }(\%) \text { positive }\end{array}$ & $\begin{array}{l}\text { Repi PLC Dairy }(n=101) \\
\text { No }(\%) \text { positive }\end{array}$ & $\begin{array}{l}\text { Yeka-Abado }(\mathrm{n}=109) \\
\text { No }(\%) \text { positive }\end{array}$ & \\
\hline Fasciola hepatic & $20(3.83)$ & $1(0.99)$ & $8(7.34)$ & $29(3.96)$ \\
\hline F. gigantica & $8(1.53)$ & - & - & $8(1.09)$ \\
\hline Trichuris sp. & $7(1.3)$ & - & $1(0.92)$ & $8(1.09)$ \\
\hline Strongyle spp. & $27(5.17)$ & $2(1.98)$ & $2(1.83)$ & $31(4.23)$ \\
\hline Coccidia & $8(1.53)$ & $2(1.98)$ & - & $10(1.37)$ \\
\hline Hydatid cyst & $21(4.1)$ & - & - & $21(2.87$ \\
\hline Mixed infections & $119(22.8)^{\mathrm{H+}}$ & - & $24(22.02)^{\mathrm{UH}}$ & $143(19.54)$ \\
\hline Total & $210(40.23)$ & $5(4.95)$ & $35(32.11)$ & $250(34.15)$ \\
\hline
\end{tabular}

Note: Mixed infection includes Fasciola spp, Strongyle spp, Emeria spp, hydatid cyst in the abattoir and Fasciola spp, Strongyle spp, Emeria spp in Yeka-Abado farmers association. $\mathrm{H}+=$ including hydatid cyst, $\mathrm{UH}=$ hydatid cyst unknown 
IHAT

In Table 2, comparisons between postmortem inspection and the IHAT results are shown, which depict $100 \%$ sensitivity and at least $90.00 \%$ specificity. No significant difference in seroprevalence rates was observed in cattle with and without gastrointestinal parasites, $\mathrm{P}>0.05$ (Tables $3 a, 3 b)$.

Table 2. Comparison of IHAT between C. bovis confirmed and negative control cattle.

\begin{tabular}{llll}
\hline & \multicolumn{2}{c}{ IHAT } & Total \\
& Positive & Negative & \\
\hline Cysticercus bovis & 39 & 0 & 39 \\
Negative control & 0 & 11 & 11 \\
Total & 39 & 11 & 50 \\
\hline
\end{tabular}

Table 3a: Comparison between IHA tests and gastrointestinal parasite infection.

\begin{tabular}{ll}
\hline Faecal examination & \multicolumn{1}{c}{ IHA } \\
& \multicolumn{1}{c}{ No(\%)positive } \\
\hline \# Positive $(\mathrm{n}=250)$ & $65(6)$ \\
\# Negative $(\mathrm{n}=482)$ & $125(25.9)$ \\
Total $(\mathrm{n}=732)$ & $190(51.8)$ \\
\hline
\end{tabular}

Note: \# Positive $(n=250)$ : Number of cattle infected with one or more gastrointestinal parasite.

\# Negative ( $n=482)$ : Number of cattle negative for gastrointestinal parasite eggs. $\mathrm{P}=0.118$.

Table $3 b$. Comparison between IHAT and gastrointestinal parasite infection in $C$. bovis confirmed cattle.

\begin{tabular}{ll}
\hline Faecal examination & IHA \\
& No(\%)positive \\
\hline \# Positive $(\mathrm{n}=19)$ & $19(100)$ \\
\# Negative $(\mathrm{n}=20)$ & $20(100)$ \\
Total $(\mathrm{n}=39)$ & $39(100)$ \\
\hline
\end{tabular}

Using IHAT, the overall prevalence of $C$. bovis in Addis Ababa abattoir was determined to be $28.5 \%$, which is 3-4 times greater than the prevalence $(7.5 \%)$ determined by the conventional method of inspection (Table 4).

The sero-prevalence of $C$. bovis infection at Repi PLC Dairy Farm and Yeka-Abado Farmers Association was $7.9 \%$ and $30.2 \%$, respectively. The difference was statistically significant $(\mathrm{P}<0.05)$. No significance difference was observed $(\mathrm{P}>0.05)$ between Yeka-Abado Farmers Association and Addis Ababa Abattoir, where the sero-prevalence was $30.3 \%$ and $28.5 \%$, respectively (Table 4 ).
Table 4: Results of IHAT using crude $C$. bovis extract on sera collected from different sources of cattle.

\begin{tabular}{ll}
\hline Source of cattle & $\begin{array}{l}\text { IHAT } \\
\text { No }(\%) \text { positive }\end{array}$ \\
\hline Abattoir $(\mathrm{n}=522)$ & $149(28.5)$ \\
Negative sera $(\mathrm{n}=11)$ & $0(0)$ \\
Repi Dairy Farm $(\mathrm{n}=101)$ & $8(7.9)$ \\
Yeka-Abado Farmers' & $33(30.3)$ \\
Association $(\mathrm{n}=109)$ & \\
& \\
Total $(\mathrm{n}=743)$ & $190(25.7)$ \\
\hline
\end{tabular}

\section{DISCUSSION}

The current study revealed that bovine cysticercosis in Ethiopia was more prevalent than hitherto believed. Previous prevalence rates in Ethiopia, based on routine meat inspection, ranged from 2.2 to 3.2\% (Tembo, 2001; Gebre Emanuel Teka, 1997). During the present study period, we found that $7.5 \%$ of cattle presented for slaughter at Addis Ababa abattoir harboured C. bovis cysts. We believe that these rates are likely to be gross underestimates, due to the inefficiency of the conventional method of inspection. Cysticerci are easily missed, as they may not be present on routine cuts considering that most cases of cysticercosis are light infections (Dorny et al., 2000). Moreover, live cysts are translucent and often pinkish in colour and may therefore be less conspicuous in the meat than dead cysts that usually form white and fibrotic lesions (OnyangoAbuje et al., 1996). To these differences in the skills and motivation of meat inspectors, the speed of the slaughter activity, and the meat inspection facilities, could be among the many other contributory factors. Therefore, the current palpation and incision techniques of routine meat inspection cannot detect the true picture of $T$. saginata Cysticercosis occurrence. Previous studies showed a 5-50 time higher prevalence rates by complete slicing of the predilection site (Minozzo et al., 2002).

In this study, using IHAT, the prevalence of $C$. bovis infection in cattle slaughtered at Addis Ababa Abattoir was determined to be $28.5 \%$. The IHAT that we have set-up showed $100 \%$ sensitivity and at least $90.00 \%$ specificity. All the 39 cattle that were positive for cysticercosis during postmortem inspection were positive by IHAT. The observed discrepancy in prevalence rates between meat inspection results $(7.5 \%)$ and IHAT $(28.5 \%)$ are not 
unexpected. Other studies in Nigeria have found meat inspection results of $3.4 \%$ and seroprevalence of $12.3 \%$ (Faleke and Ogundipe, 2004). The test could be sensitive to circulating antibodies where the cyst has already calcified.

The sero-prevalence of bovine cysticercosis in Repi PLC Dairy Farm and Yeka-Abado Farmers Association was determined to be $7.9 \%$ and $30.2 \%$, respectively, and this difference was statistically significant $(\mathrm{P}<0.05)$. Not surprisingly, the seroprevalence rate in Addis Ababa Abattoir (28.5\%) is comparable with that of Yeka-Abado Farmers Association with prevalence of $30.3 \%$ ( $\mathrm{P}>0.05$ ). The Repi PLC Dairy Farm is relatively well managed and employs permanent personnel that are living in the farm premises with relatively good hygienic conditions. In this farm, replacement stock is usually bred, which can be expected to reduce the risk of infection. Furthermore, de-worming is done every four months, and the animals are mostly kept indoors. This difference in prevalence between Yeka-Abado Farmers Association and Repi PLC Dairy Farm was likely to be due to differences in management. The use of causal workers in feedlots is a factor that is conductive to T. saginata transmission (Dorny et al., 2002). The difference in farm management is also clearly depicted by the low prevalence $(3.5 \%)$ of gastrointestinal helminthiases in Repi PLC Dairy Farm, which contrasts with a high rate of $32.1 \%$ in Yeka-Abado Farmers Association. In addition, the management practices exercised in dairy and extensive farms is different due to the source of replacement stocks. In Repi PLC dairy farm replacement stock is bred within the farm and while in the extensive farm, Yeka-Abado Farmers Association, grazing and watering sites are communal. There are no latrines in the area, bush defecation is practiced and animals often get in contact with human faeces.

In order to reduce the risk of human infection there is an urgent need to assessing the risk factors of infection in cattle. The IHAT can be a useful tool for sero-epidemiological surveys and can be conducted on live herds. It may be considered as part of an integrated quality control programs for determining the quality of beef products and for developing standards of hygiene. The test is relatively cheap and can be performed in most laboratories, as it does not require sophisticated equipment. Furthermore, even though circumstantial, we found minimal cross-reactions with other helminthes. Given the high levels of sensitivity and more than $92.74 \%$ specificity, the IHAT that we have developed can be used as a diagnostic test for epidemiological surveys to estimate the national prevalence of the disease. With further improvements and evaluation, the test could be used to screen animals for international movement, and to provide a degree of confidence on the predictive value of a negative test. In order to increase the diagnostic potential of the IHAT, further refinement and improvement of the test system is recommended.

\section{ACKNOWLEDGEMENTS}

We are grateful to Prof. P. Dorchies for the provision of negative control sera. We thank Dr. Bitew Getahun, head of meat inspection team and the meat inspectors for their cooperation while collecting samples at Addis Ababa Abattoir. Drs Fekadu Kebede and Lakemariam Yigezu are thanked for providing us with the necessary reagents and laboratory supplies. Dr Wondwosen Tsegay, Shola Regional Laboratory Research Officer, is also thanked for his unreserved cooperation during collection of samples at Repi PLC Dairy Farm and Yeka-abado Farmers Association. Asseged Kassa and Bethelhem Tefera, both from Faculty of Medicine; and Demeku Nega, Woinishet Mekonnen, Baysasu G/Medhin, Nega Nigussie, Hailu Getu and Abebe Animut from Aklilu Lemma Institute of Pathobiology are greatly acknowledged for their contribution in sample collection and laboratory assistance. The Core Laboratory of Faculty of Medicine and the Aklilu Lemma Institute of Pathobiology are acknowledged for allowing their facilities. The study was funded by the School of Graduate Studies, Addis Ababa University.

\section{REFERENCES}

1. Cabaret, J., Geerts, S., Madeline, M., Ballandonne, C. and Barbier, D. (2002). The use of urban sewage sludge on pastures: The cysticercosis threat. Vet. Res. 33: 575-597.

2. Dorny, P., Phiri, I., Gabriel, S., Speybroeck, N. And Vercruysse, J. (2002). A Sero-epidemiological study of bovine cystcercosis in Zambia. Vet. Parasitol. 104(3):211-215.

3. Dorny, P., Vercammen, F., Brandt, J., Vansteenkiste, W., Berkvens, D. and Geerts, S. (2000). SeroEpidemiological Study of Taenia saginata Cysticercosis in Belgian Cattle. Vet. Parasitol., 88:43-49.

4. EARO (2000). Beef Research Strategy. Ethiopian Agricultural Research Organization (EARO), Animal Science Directorate, Addis Ababa, pp. $1-16$. 
5. Faleke, O.O. and Ogundipe, G.A. (2004). Taenia saginata cysticercosis in Oyo State, Nigeria. Trop Vet. 22(3\&4):113-117.

6. Ferreira, A.P., Vaz, A.J., Nakamura, P.M., Sasaki, A.T., Ferreira, A.W. and Livramento, J.A. (1997). Hemagglutination test for the diagnosis of human neurocysticercosis: development of a stable reagent using homologous and heterologous antigens. Rev. Inst. Med. Trop. S. Paulo 39(1):29-36.

7. Hancock, D.D., Swikse, E. and Lichtenwalner, A.B. (1989). Distribution of bovine cysticercosis in Washington. Am. J. Vet. Res. 50:564-570.

8. Kaufmann, J. (1998). Parasite Infections of Domestic Animals. A Diagnostic Manual. Birkhauser, pp. 1-143.

9. Lowry, O.H., Rosebrough, N.J., Farr, A.L. and Randall, R.J. (1951). Protein measurement with the Folin phenol reagent. J Biol Chem, 193:265275.

10. MoA (1972). Meat inspection regulations. Legal Notice No-428. Negarit Gazeta. Ministry of Agriculture (MoA), Addis Ababa, Ethiopia.

11. Minozzo, J.C., Gusso, R.L.F., De Castro, E.A., Lago, O. and Soccoi, V.T. (2002). Experimental bovine infection with Taenia saginata eggs: recovery rates and cysticerci location. Braz. Arch. Biol. Technol. 45(4): 451-455.

12. Onyango-Abuje, J.A, Hughes, G., Opicha, M., Niginyi, K.M., Rugutt, M.K., Wrightand, S.H., and Harrison, L.J.S. (1996). Diagnosis of Taenia saginata Cystcercosis in Kenian Cattle by Antibody and Antigen E.L.I.S.A. Vet. Parasitol. 61(8):221-230.

13. Shirai A., Dietel J.W. and Osterman J.V. (1975). Indirect hemagglutination test for human antibody to typhus and spotted fever group rickettsiae. J Clinic Microbiol., 2(5):430-437.

14. Sloss, M.W., Kemp, R.L. and Zajac, A.M. (1994). Veterinary Clinical Parasitology, $6^{\text {th }}$ ed. Iowa State Press. A Blackwell Publishing Company, pp. 3100.

15. Gebre Emanuel Teka. (1997). Food Hygiene Principles and Food Borne Disease Control with Special Reference to Ethiopia, $1^{\text {st }}$ ed. Department of Community Health, Faculty of Medicine, Addis Ababa University, pp. 40-62.

16. Tembo A, (2001). Epidemiology of Taenia saginata, Taeniasis/ Cysticercosis in three Selected agroclimatic zones. Faculty of Veterinary Medicine, Addis Ababa University, Debre Zeit and Free University of Berlin, Berlin, MSc Thesis, pp, 1150.

17. Thrusfield, M. (1995). Veterinary Epidemiology. $2^{\text {nd }}$ ed. Blackwell Science, pp. 266-285.

18. Yamamoto, Y.I., Hoshino-Shimizu, S. and Camargo, M.E. (1991). A novel IgM-indirect hemagglutination test for the serodiagnosis of acute Toxoplasmosis. J. Clin. Lab. Anal. 5:127132. 